\title{
On Transformations of Distribution Functions on the Unit Interval - a Generalization of the Gauß-Kuzmin-Lévy Theorem
}

\author{
P. SchatTE
}

Let $X$ be a random variable on $[0,1)$ with the distribution function $g_{0}$ and $h$ be a piecewise monotonic transformation on [0,1). Further denote the distribution function of $h(X)$ by $g_{1}$, of $h(h(X))$ by $g_{2}$, and so on. The distribution functions $g_{0}, g_{1}, g_{2}, \ldots$ can also be regarded as the asymptotic distribution functions of sequences $\left(a_{n}\right),\left(h\left(a_{n}\right)\right),\left(h\left(h\left(a_{n}\right)\right)\right), \ldots$ The operator $P_{h}$ defined by $g_{n+1}^{\prime}=P_{h} g_{n}^{\prime}$ is the so-called Frobenius-Perron operator assigned to the transformation $h$. We say that $h$ preserves the distribution function $k$ if $g_{0}=k$ implies $g_{1}=k$ and consequently $g_{n}=k$ for all $n$, i.e., if $k^{\prime}$ is an invariant element of the operator $P_{h}$. Then under weak additional suppositions $g_{n}^{(r)}=k^{(r)}+O\left(q^{n}\right)$ for $r=0,1,2$ and for arbitrary initial distribution functions $g_{0}$, where $q<1$ depends on $h$ but not on $g_{0}$. This result generalizes the Gauss-Kuzmin-Lévy theorem in the metric theory of continued fractions. Particularly, piecewise linear transformations preserve the uniform distribution. In this case more precise estimates are possible under weaker suppositions.

Key words: Piecewise monotonic transformations, Frobenius-Perron operators, invariant measures, uniform distribution of sequences, metric theory of continued fractions

AMS subject classification: $58 \mathrm{~F} 11,28 \mathrm{D} 05,11 \mathrm{~K} 06,11 \mathrm{~K} 50$

\section{Introduction}

Recently, Bosch [1] and Porubský, Śalát, and Strauch [14] have studied transformations $h$ that preserve the uniform distribution of sequences. On the other hand, Goh and Schmutz [2] have discovered that high iterates of certain piecewise monotonic transformations $h$ generate the uniform distribution even if the initial distribution is not uniform. In what follows we wish to unify both directions of research. We shall show that piecewise monotonic transformations $h$ that preserve the uniform distribution generate also the uniform distribution in the limit if high iterates are applied to an arbitrary initial distribution function. The preserving transformation $h$ must only satisfy weak additional assumptions. The rate of convergence is estimated to be geometric. The best estimates are possible, of course, in the case of piecewise linear transformations.

All assertions can be extended to the case of piecewise monotonic transformations $h$ which preserve a more general distribution function $k$. For instance, the transformation $h(x)=\{1 / x\}$ preserves the distribution function $k(x)=\log (1+x) / \log 2$. This transformation is considered in the theory of continued fractions, cf., e.g., Khintchine [10], and is the subject of the celebrated Gauss-Kuzmin-Lévy theorem which is now contained in our estimates as a special case.

The transformation $h$ of the unit interval induces a transformation $P_{h}$ of probability densities on the unit interval. This transformation is accomplished by the so-called Frobenius-Perron operator assigned to $h, \mathrm{cf}$. the remark succeeding Theorem 1 . The convergence of iterates of the Frobenius-Perron operator is studied by, e.g., Lasota and Yorke $[12,13]$, Hofbauer and Keller [3], Jabłoński, Kowalski, and Malczak [6 - 9]. Our results are especially related to those in [6 9] on the geometric convergence of iterates of the Frobenius-Perron operator. However, all the above mentioned authors consider only finite partitions of the unit interval $[0,1)$ and their results

P. Schatte: Bergakademie Freiberg, FB Mathematik, B.-Cotta-Str. 2, D - 09599 Freiberg 
are especially not applicable to the Gauss-Kuzmin-Lévy theorem. See for example also Ishitani [5], who has considered infinite partitions in another connection. In case of finite partitions our suppositions are somewhat different from those in [6 - 9].

\section{Transformations}

We partition the unit interval $[0,1)$ into a denumerable set of subintervals $\left[x_{i}, y_{i}\right)$ such that

$$
[0,1)=\bigcup_{i=1}^{\infty}\left[x_{i}, y_{i}\right)
$$

Let $h_{i} \in C^{2}[0,1)$ be given for $i=1,2, \ldots$. We assume the $h_{i}$ increasing on $[0,1)$ and suppose $h_{i}(0)=x_{i}, h_{i}(1-0)=y_{i}$. Then we define the piecewise monotonic transformation $h$ by $h(x)=$ $h_{i}^{-1}(x)$ on $\left[x_{i}, y_{i}\right)$.

We do not assume that $x_{i+1}=y_{i}$ or that $x_{i}=y_{i+1}$. Thus we permit an infinite number of limit points of the $x_{i}$ in the unit interval. The case of $y_{i}=x_{i}$ for $i>N$ is included. Thus we consider also finite partitions of $[0,1)$.

Furthermore we mention that some or all $h_{i}$ can be decreasing with $h_{i}(0)=y_{i}, h_{i}(1-0)=x_{i}$. Then the following considerations must be modified in an obvious manner. But for the sake of transparence we suppress the explicit inclusion of this generalization.

Theorem 1 : Let $X$ be a random variable on $[0,1)$ with the continuous distribution function $g_{0}(x)=\mathcal{P}(X<x)$. Then $h(X)$ has the distribution function

$$
g_{1}(x)=\sum_{i=1}^{\infty}\left(g_{0}\left(h_{i}(x)\right)-g_{0}\left(x_{i}\right)\right)
$$

Furthermore, if $g_{0}$ is absolutely continuous, $\left|g_{0}^{\prime}(x)\right| \leq C$ a.e. and if $\sum_{i=1}^{\infty} h_{i}^{\prime}(x)$ converges a.e. in $[0,1)$ to an integrable function, then $g_{1}$ is also absolutely continuous and

$$
g_{1}^{\prime}(x)=\sum_{i=1}^{\infty} g_{0}^{\prime}\left(h_{i}(x)\right) h_{i}^{\prime}(x) \text { a.e. in }[0,1)
$$

Proof: By the theorem of total probability we have

$$
\mathcal{P}(h(X)<x)=\sum_{i=1}^{\infty} \mathcal{P}\left(h(X)<x, x_{i} \leq X<y_{i}\right)
$$

which proves (2). Now the right-hand side of (3) converges a.e. to an integrable function, say $g$. Then we see by the dominated convergence theorem that

$$
\int_{0}^{x} g(t) \ddot{d t}=\sum_{i=1}^{\infty} \int_{0}^{x} g_{0}^{\prime}\left(h_{i}(t)\right) h_{i}^{\prime}(t) d t=\sum_{i=1}^{\infty}\left(g_{0}\left(h_{i}(x)\right)-g_{0}\left(x_{i}\right)\right)=g_{1}(x)
$$

which proves (3)

Remark: By (3) the initial probability density $g_{0}^{\prime}$ is transformed into the probability density $g_{1}^{\prime}$. This transformation defines the Frobenius-Perron operator $P_{h}$ by $g_{1}^{\prime}=P_{h} g_{0}^{\prime}$.

Let $\left(a_{k}\right)_{k=1}^{\infty}$ be a sequence of real numbers. The sequence $\left(a_{k}\right)$ is said to have the asymptotic distribution function $g_{0}$ if

$$
\lim _{K \rightarrow \infty} \frac{1}{K} \sum_{k=1}^{K} 1_{[0, x)}\left(\left\{a_{k}\right\}\right)=g_{0}(x)
$$


for $x \in[0,1)$, cf. Kuipers and Niederreiter [11: p.53]. Here $1_{(0, x)}$ is the indicator function of the interval $[0, x)$ and $\left\{a_{k}\right\}$ is the fractional part of $a_{k}$. Since only the fractional parts of $a_{k}$ are of interest, we suppose in the following that $0 \leq a_{k}<1$.

Theorem 2: If the sequence $\left(a_{k}\right)_{k=1}^{\infty}$ has the asymptotic distribution function $g_{0}$ which is assumed to be absolutely continuous, then the sequence $\left(h\left(a_{k}\right)\right)_{k=1}^{\infty}$ has the asymptotic distribution function $g_{1}$ given by (2).

Proof: We choose $N$ so large that $\sum_{i=1}^{N}\left(g_{0}\left(y_{i}\right)-g_{0}\left(x_{i}\right)\right)>1-\epsilon$. This is possible on account of the absolute continuity of $g_{0}$. Then we have

$$
\begin{aligned}
\sum_{i=1}^{N} \frac{1}{K} \sum_{k=1}^{K} 1_{\left[x_{i}, h_{i}(x)\right)}\left(a_{k}\right) & \leq \frac{1}{K} \sum_{k=1}^{K} 1_{[0, x)}\left(h\left(a_{k}\right)\right) \\
& \leq \sum_{i=1}^{N} \frac{1}{K} \sum_{k=1}^{K} 1_{\left[x_{i}, h_{i}(x)\right)}\left(a_{k}\right)+1-\sum_{i=1}^{N} \frac{1}{K} \sum_{k=1}^{K} 1_{\left[x_{i}, y_{i}\right)}\left(a_{k}\right) .
\end{aligned}
$$

Letting $K \rightarrow \infty$ we obtain

$$
\begin{aligned}
\sum_{i=1}^{N}\left(g_{0}\left(h_{i}(x)\right)-g_{0}\left(x_{i}\right)\right) & \leq \liminf _{K \rightarrow \infty} \frac{1}{K} \sum_{k=1}^{K} 1_{[0, x)}\left(h\left(a_{k}\right)\right) \\
& \leq \limsup _{K \rightarrow \infty} \frac{1}{K} \sum_{k=1}^{K} 1_{[0, x)}\left(h\left(a_{k}\right)\right) \leq \sum_{i=1}^{N}\left(g_{0}\left(h_{i}(x)\right)-g_{0}\left(x_{i}\right)\right)+\epsilon
\end{aligned}
$$

which proves the theorem for $\epsilon \rightarrow 0$ I

Definition : We say that a transformation $h$ preserves the distribution function $k$ if $g_{0}=k$ is transformed by (2) into $g_{1}=k$.

The transformation $h$ preserves the distribution function $k$ if the probability density $k^{\prime}$ is an invariant element of the Frobenius-Perron operator $P_{h}$. Particularly, the transformation $h$ preserves the uniform distribution if 1 is an invariant element of $P_{h}$.

Theorem 3: The transformation $h$ preserves the uniform distribution if and only if

$$
\sum_{i=1}^{\infty}\left(h_{i}(x)-x_{i}\right)=x
$$

for $x \in[0,1)$. Furthermore, if

$$
\sum_{i=1}^{\infty} h_{i}^{\prime}(x)=1 \text { a.e. in }[0,1)
$$

then $h$ preserves the uniform distribution.

Proof: The condition (4) follows from (2) by definition. Let (5) be satisfied. Then by the monotone convergence theorem and by $h_{i}(0)=x_{i}$ we have

$$
x=\int_{0}^{x} \sum_{i=1}^{\infty} h_{i}^{\prime}(t) d t=\sum_{i=1}^{\infty} \int_{0}^{x} h_{i}^{\prime}(t) d t=\sum_{i=1}^{\infty}\left(h_{i}(x)-x_{i}\right) .
$$

Thus (4) is satisfied I

Theorem 3 is related to Theorem 2.9 in Bosch [1]. Obviously (5) implies that $h_{i}^{\prime}(x) \leq 1$ on $[0,1)$ for $i=1,2, \ldots$ 


\section{Higher iterates}

We define recursively the random variables $X_{n}$ by $X_{n+1}=h\left(X_{n}\right), X_{0}=X$, the sequences $\left(a_{k, n}\right)$ by $a_{k, n+1}=h\left(a_{k, n}\right), a_{k, 0}=a_{k}$, and the distribution functions $g_{n}$ by

$$
g_{n+1}(x)=\sum_{i=1}^{\infty}\left(g_{n}\left(h_{i}(x)\right)-g_{n}\left(h_{i}(0)\right)\right) .
$$

In order to obtain $g_{n}$ directly from $g_{0}$, we introduce the vector $j_{n}=\left(i_{1}, i_{2}, \ldots, i_{n}\right)$ and put

$$
h\left(j_{n+1} ; x\right)=h\left(j_{n} ; h_{i_{n+1}}(x)\right), h\left(j_{1} ; x\right)=h_{i_{1}}(x) .
$$

Corollary 4 : We have

$$
g_{n}(x)=\sum_{j_{n}}\left(g_{0}\left(h\left(j_{n} ; x\right)\right)-g_{0}\left(h\left(j_{n} ; 0\right)\right)\right)
$$

Proof: We apply induction on $n$. For $n=1$ the equality (8) is identical with (2). From (6) and (8) we obtain

$$
\begin{aligned}
g_{n+1}(x)= & \sum_{i=1}^{\infty} \sum_{j_{n}}\left(g_{0}\left(h\left(j_{n} ; h_{i}(x)\right)\right)-g_{0}\left(h\left(j_{n} ; 0\right)\right)\right) \\
& -\sum_{i=1}^{\infty} \sum_{j_{n}}\left(g_{0}\left(h\left(j_{n} ; h_{i}(0)\right)\right)-g_{0}\left(h\left(j_{n} ; 0\right)\right)\right) \\
= & \sum_{j_{n+1}}\left(g_{0}\left(h\left(j_{n+1} ; x\right)\right)-g_{0}\left(h\left(j_{n+1} ; 0\right)\right)\right)
\end{aligned}
$$

by (7). This is (8) with $n$ replaced by $n+1$

Corollary $5:$ If $g_{0}$ is the distribution function of $X$, then $g_{n}$ is the distribution function of $X_{n}$. If $g_{0}$ is absolutely continuous and the asymptotic distribution function of the sequence $\left(a_{k}\right)_{k=1}^{\infty}$, then $g_{n}$ is the asymptotic distribution function of the sequence $\left(a_{k, n}\right)_{k=1}^{\infty}$ :

Corollary 6: If the transformation $h$ preserves the uniform distribution, then it follows that $\sum_{j_{n}}\left(h\left(j_{n} ; x\right)-h\left(j_{n} ; 0\right)\right)=x$.

Proof: Put $g_{0}(x)=g_{n}(x)=x$ in (8) I

Lemma 7 : If $(5)$ holds a.e. in $[0,1)$, then

$$
\sum_{j_{n}} h^{\prime}\left(j_{n} ; x\right)=1 \text { a.e. in }[0,1) \text {. }
$$

Furthermore, if $g_{0}$ is absolutely continuous, $\left|g_{0}^{\prime}\right| \leq C$ a.e., then $g_{n}$ is absolutely continuous and

$$
g_{n}^{\prime}(x)=\sum_{j_{n}} h^{\prime}\left(j_{n} ; x\right) g_{0}^{\prime}\left(h\left(j_{n} ; x\right)\right) \text { a.e. in }[0,1) .
$$

Proof: The equality (9) will be proved by induction on $n$. Namely,

$$
\sum_{j_{n+1}} h^{\prime}\left(j_{n+1} ; x\right)=\sum_{i_{n+1}} \sum_{j_{n}} h^{\prime}\left(j_{n} ; h_{i_{n+1}}(x)\right) h_{i_{n+1}}^{\prime}(x)
$$


on account of (7). Now (10) can be concluded from (8) in the same way as (3) was concluded from (2) in the proof of Theorem 1 I

Remark : Corollaries 4-6 and Lemma 7 can be summarized to the assertion that the transformation $h^{\circ n}=h \circ \ldots \circ h$ is given by the $h\left(j_{n} ; x\right)$ and satisfies the same suppositions as $h$.

Lemma 8 : Assume that (5) holds a.e. in $[0,1)$. Further let
a) $h_{i}^{\prime}(x) \leq d<1$
b) $\left|h_{i}^{\prime \prime}(x)\right| \leq K h_{i}^{\prime}(x)$,

for $x \in[0,1)$ and $i=1,2, \ldots$ If moreover $g_{0}(x) \in C^{2}[0,1]$, i.e. , if
a) $\left|g_{0}^{\prime}(x)-1\right| \leq C_{1}$
b) $\left|g_{0}^{\prime \prime}(x)\right| \leq C_{2}$,

then $g_{n}^{\prime \prime} \in C^{2}[0,1]$ too, and
a) $\left|g_{1}^{\prime \prime}(x)\right| \leq K C_{1}+d C_{2}$
b) $\left|g_{n}^{\prime \prime}(x)\right| \leq K C_{1} /(1-d)+d^{n} C_{2}, \quad n \geq 2$.

Proof: From (7) it follows that

$$
h^{\prime}\left(j_{n} ; x\right) \leq d^{n} .
$$

Further we show by induction on $n$ that

$$
\left|h^{\prime \prime}\left(j_{n} ; x\right)\right| \leq K h^{\prime}\left(j_{n} ; x\right) /(1-d) .
$$

Namely, from (7) we conclude that

$$
\begin{aligned}
\left|h^{\prime \prime}\left(j_{n+1} ; x\right)\right| & =\left|h^{\prime \prime}\left(j_{n} ; h_{i_{n+1}}(x)\right) h_{i_{n+1}}^{2}(x)+h^{\prime}\left(j_{n}, h_{i_{n+1}}(x)\right) h_{i_{n+1}}^{\prime \prime}(x)\right| \\
& \leq K h^{\prime}\left(j_{n} ; h_{i_{n+1}}(x)\right) h_{i_{n+1}}^{\prime}(x) d /(1-d)+K h^{\prime}\left(j_{n} ; h_{i_{n+1}}(x)\right) h_{i_{n+1}}^{\prime}(x) \\
& \leq K h^{\prime}\left(j_{n+1} ; x\right) /(1-d) .
\end{aligned}
$$

From (15) we derive,

$$
\begin{aligned}
& \left|\log h^{\prime}\left(j_{n} ; x\right)-\log h^{\prime}\left(j_{n} ; y\right)\right| \leq|x-y|\left|h^{\prime \prime}\left(j_{n} ; \xi\right)\right| / h^{\prime}\left(j_{n} ; \xi\right) \leq K /(1-d) \\
& h^{\prime}\left(j_{n} ; x\right) / h^{\prime}\left(j_{n} ; y\right) \leq L
\end{aligned}
$$

for arbitrary $x, y \in[0,1)$. Now we see that (9) holds uniformly in $x$. By termwise differentiation we get

$$
\sum_{j_{n}} h^{\prime \prime}\left(j_{n} ; x\right)=0, \quad g_{n}^{\prime \prime}(x)=\sum_{j_{n}} h^{\prime \prime}\left(j_{n} ; x\right)\left(g_{0}^{\prime}\left(h\left(j_{n} ; x\right)\right)-1\right)+\sum_{j_{n}} h^{\prime 2}\left(j_{n} ; x\right) g_{0}^{\prime \prime}\left(h\left(j_{n} ; x\right)\right)
$$

also uniformly in $[0,1)$ such that $(13)$ follows

\section{Asymptotic estimates}

In the following we wish to show the geometric convergence of the $g_{n}$ to the uniform distribution or to a more general distribution function $k$ which is preserved under the piecewise monotonic transformation $h$ given by the $h_{i}$.

Theorem 9 : Let $g_{0} \in C^{2}[0,1]$ and assume that (5) and (11) and are satisfied. Then there exists a real $q<1$ such that
a) $g_{n}^{\prime}(x)=1+O\left(q^{n}\right)$
b) $g_{n}^{\prime \prime}(x)=O\left(q^{n}\right)$ 
Proof: From (16) we get $\Delta_{j_{n}}=h\left(j_{n} ; 1\right)-h\left(j_{n} ; 0\right)=h^{\prime}\left(j_{n} ; \xi_{j_{n}}\right) \leq L h^{\prime}\left(j_{n} ; x\right)$. By Lemma 7 we have

$$
\begin{aligned}
g_{n}^{\prime}(x)-1= & \sum_{j_{n}} h^{\prime}\left(j_{n} ; x\right)\left(g_{0}^{\prime}\left(h\left(j_{n} ; x\right)\right)-1\right) \\
= & \sum_{j_{n}}\left(h^{\prime}\left(j_{n} ; x\right)-\Delta_{j_{n}} / L\right)\left(g_{0}^{\prime}\left(h\left(j_{n} ; x\right)\right)-1\right) \\
& +\frac{1}{L} \sum_{j_{n}}\left(\Delta_{j_{n}}\left(g_{0}^{\prime}\left(h\left(j_{n} ; x\right)\right)-1\right)-\int_{h\left(j_{n} ; 0\right)}^{h\left(j_{n} ; 1\right)}\left(g_{0}^{\prime}(x)-1\right) d x\right)
\end{aligned}
$$

where the equalities

$$
\sum_{j_{n}} \Delta_{j_{n}}=1, \sum_{j_{n}} \int_{h\left(j_{n} ; 0\right)}^{h\left(j_{n} ; 1\right)}\left(g_{0}^{\prime}(x)-1\right) d x=\int_{0}^{1}\left(g_{0}^{\prime}(x)-1\right) d x=0
$$

are used. The first one can be proved by induction on $n$ whereas the second one is a consequence of the first one. Since $g_{0} \in C^{2}[0,1]$, condition (12) is fulfilled. Thus we obtain

$$
\begin{aligned}
\left|g_{n}^{\prime}(x)-1\right| & \leq \sum_{j_{n}}\left(h^{\prime}\left(j_{n} ; x\right)-\Delta_{j_{n}} / L\right) C_{1}+\left|\frac{1}{L} \sum_{j_{n}} \Delta_{j_{n}}\left(g_{0}^{\prime}\left(h\left(j_{n} ; x\right)\right)-g_{0}^{\prime}\left(h\left(j_{n} ; \tau_{j_{n}}\right)\right)\right)\right| \\
& \leq(1-1 / L) C_{1}+\left|\frac{1}{L} \sum_{j_{n}} \Delta_{j_{n}}\left(x-\tau_{j_{n}}\right) g_{0}^{\prime \prime}\left(h\left(j_{n} ; \sigma_{j_{n}}\right)\right) h^{\prime}\left(j_{n} ; \sigma_{j_{n}}\right)\right| \\
& \leq(1-1 / L) C_{1}+d^{n} C_{2} / L,
\end{aligned}
$$

by (14). On the other hand, from Lemma 8 we get $\left|g_{n}^{\prime \prime}(x)\right| \leq K C_{1} /(1-d)+d^{n} C_{2}$. Now we choose $l$ so large that $d^{l} \leq \min ((1-d) / 6 K, 1 / 6)$. Moreover we suppose $K>0$ and enlarge $C_{1}$ or $C_{2}$ such that $C_{2}=3 K C_{1} /(1-d)$. Then

$$
=\left|g_{l}^{\prime}(x)-1\right| \leq(1-1 / 2 L) C_{1} \text { and }\left|g_{l}^{\prime \prime}(x)\right| \leq 3 K(1-1 / 2 L) C_{1} /(1-d) .
$$

Starting with $g_{k}$ instead of $g_{0}$ we arrive at

$$
\left|g_{m l+k}^{\prime}(x)-1\right| \leq(1-1 / 2 L)^{m} C \quad \text { and } \quad\left|g_{m l+k}^{\prime \prime}(x)\right| \leq 3 K(1-1 / 2 L)^{m} C /(1-d)
$$

for $0 \leq k<n, m=0,1, \ldots$, and $C=\sup _{x \in[0,1], 0 \leq k<l}\left(\left|g_{k}^{\prime}(x)-1\right|,\left|g_{k}^{\prime \prime}(\dot{x})\right|(1-d) / 3 K\right)$ |

Note that $q=(1-1 / 2 L)^{1 / l}$ depends on $h$ but not on $g_{0}$. Only $C$ depends on $g_{0}$. If $\left|h^{\prime}(x)\right| \leq M$ for $x \in[0,1]$, then $h_{i}^{\prime}(x) \geq 1 / M$, and the supposition (11)/b) will be satisfied in many cases because $h_{i} \in C^{2}[0,1)$. However, $\left|h^{\prime}(x)\right| \leq M$ can only hold for finite partitions of the unit interval. The supposition (11)/a) will also be satisfied in many cases since $h_{i}^{\prime}(x) \leq 1$. is implied by (5). Thus the condition (5) is the most significant supposition for Theorem 9 , it ensures that $h$ preserves the uniform distribution.

We remark that the supposition $g_{0}, h_{i} \in C^{2}[0,1]$ can be replaced by a Lipschitz condition for $g_{0}^{\prime}$ and $h_{i}^{\prime}$.

Now we assume that the transformation $h$ preserves the distribution function $\ddot{k}=k(x)$, where $k \in C^{2}[0,1]$ and

$$
k^{\prime}(x) \geq \delta>0
$$


for $x \in[0,1]$. Then by (2)

$$
k(x)=\sum_{i=1}^{\infty}\left(k\left(h_{i}(x)\right)-k\left(x_{i}\right)\right) .
$$

We introduce the transformation $h^{*}=k \circ h \circ k^{-1}$ by $h_{i}^{*}(x)=k\left(h_{i}\left(k^{-1}(x)\right)\right)$. In view of (19) $h_{i}^{*}$ fulfils (4), i.e., $h^{*}$ preserves the uniform distribution. Since $h=k^{-1} \circ h^{*} \circ k$, we have $h^{\circ n}=k^{-1} \circ\left(h^{*}\right)^{\circ n} \circ k$. The function $k$ transforms the initial distribution function $g_{0}$ into $g_{0}^{*}=g_{0} \circ k^{-1}$. On account of (18), the distribution function $g_{0}^{*}$ fulfils the suppositions of Theorem 9 in the same way as $g_{0}$. The distribution function $g_{0}^{*}$ is transformed by $\left(h^{*}\right)^{\circ n}$ into $g_{n}^{*}$. For $g_{n}^{*}$ the estimates (17) hold true. Finally $k^{-1}$ transforms into $g_{n}=g_{n}^{*} \circ k$.

Corollary 10: Let the transformation $h$ preserve the distribution function $k \in C^{2}[0,1]$ with (18). Assume that $h^{*}=k \circ h \circ k^{-1}$ satisfies the suppositions of Theorem 9. Furthermore let $g_{0} \in C^{2}[0,1]$. Then
a) $g_{n}(x)=k(x)+O\left(q^{n}\right)$
b) $g_{n}^{\prime}(x)=k^{\prime}(x)+O\left(q^{n}\right)$
c) $g_{n}^{\prime \prime}(x)=k^{\prime \prime}(x)+O\left(q^{n}\right)$

uniformly for $x \in[0,1]$.

Notice that the last estimate in (20) implies both others since $\int_{0}^{1} g_{n}^{\prime}(x) d x=\int_{0}^{1} k^{\prime}(x) d x=1$, $g_{n}(0)=k(0)=0$.

Corollary 10 is related to results by Jabłoński, Kowalski, and Malczak [7, 8]. But these authors consider only finite partitions of the unit interval. Moreover, in [8] they assume the $h_{i}$ to be convex, and in [7] they suppose that the $h_{i}$ satisfy certain complicated conditions which are quite different from our suppositions. On the other hand, in $[7,8]$ the initial probability density $g_{0}^{\prime}$ is required only to be of bounded variation.

Theorem 11 : Let the transformation $h$ preserve the distribution function $k \in C^{2}[0,1)$ with (18). Assume that $h^{*}=k \circ h \circ k^{-1}$ satisfies the suppositions (5) and (11). Furthermore, let $g_{0} \in C^{2}[0,1]$. Then (20) holds with $q \leq K / 2+d$.

Proof: Let $g_{0}^{*}$ satisfy (12)/b). Then we conclude that

$$
\left|g_{0}^{* \prime}(x)-1\right|=\left|\int_{0}^{1}\left(g_{0}^{* \prime}(x)-g_{0}^{* \prime}(t)\right) d t\right| \leq \int_{0}^{1}\left|g_{0}^{* \prime}(x)-g_{0}^{* \prime}(t)\right| d t \leq \int_{0}^{1} \cdot|x-t| C_{2} d t \leq C_{2} / 2
$$

and from (13)/a) that $\left|g_{1}^{* \prime \prime}(x)\right| \leq K C_{2} / 2+d C_{2}=(K / 2+d) C_{2}$. Now (20) can be obtained by iterating the given estimates

We remark that more precisely $\left|g_{n}^{* \prime \prime}(x)\right| \leq C_{2} q^{n}$ and $\left|g_{n}^{* \prime}(x)-1\right| \leq C_{2} q^{n} / 2$ are derived in the proof of Theorem 11. Further we obtain

$$
\left|g_{n}^{*}(x)-x\right| \leq \min (x, 1-x) C_{2} q^{n} / 2 \leq x(1-x) C_{2} q^{n} .
$$

In all these estimates $C_{2}$ is an upper bound for the absolute value of the second derivative of $g_{0}^{*}=g_{0} \circ k^{-1}$. For $g_{n}$ we obtain more precisely the estimates
a) $\left|g_{n}(x)-k(x)\right| \leq k(x)(1-k(x)) C_{2} q^{n}$
b) $\quad\left|g_{n}^{\prime}(x)-k^{\prime}(x)\right| \leq k^{\prime}(x) C_{2} q^{n} / 2$
c) $\left|g_{n}^{\prime \prime}(x)-k^{\prime \prime}(x)\right| \leq\left(\left|k^{\prime \prime}(x)\right| / 2+k^{\prime 2}(x)\right) C_{2} q^{n}$. 
Theorem 11 is an improvement of a result by Jabloniski and Malczak $[6,9]$ who have essentially estimated $q$ by $K+d$, again only in the case of finite partitions and for $g_{0}^{\prime}$ of bounded variation.

Example 12: We consider the transformation $h(x)=\{1 / x\}$ which plays an important role in the theory of continued fractions. It can be given by $h_{i}(x)=1 /(x+i), x_{i}=1 / i, y_{i}=1 /(1+i)$ and leads to the recurrence

$$
g_{n+1}(x)=\sum_{i=1}^{\infty}\left(g_{n}\left(\frac{1}{i}\right)-g_{n}\left(\frac{1}{x+i}\right)\right) .
$$

Since the $h_{i}$ are decreasing, the sign here is opposite to that in (6). The transformation $h$ preserves the distribution function $k(x)=\log (1+x) / \log 2$, i.e., the transformation $h^{*}$ given by

$$
h_{i}^{*}(x)=\frac{1}{\log 2}(\log (i+t)-\log (i-1+t)), \quad t=e^{x \log 2},
$$

preserves the uniform distribution. We have

$$
\begin{aligned}
& h_{i}^{* \prime}(x)=-t /(i+t)(i-1+t), \\
& h_{i}^{* \prime \prime}(x) / h_{i}^{* \prime}(x)=\log 2 \cdot\left(i(i-1)-t^{2}\right) /(i+t)(i-1+t) .
\end{aligned}
$$

The supposition (5) is satisfied in the modified form $\sum h_{i}^{* \prime}(x)=-1$, observing that the $h_{i}^{*}$ are decreasing. The supposition (11)/a) holds with $d=1 / 2,(11) / \mathrm{b}$ ) holds with $K=\log 2$. The supposition (18) for $k$ holds with $\delta=1 / 2 \log 2$. Thus (21) is valid for $q \leq(\log 2+1) / 2$ $<0.85$ if $g_{0} \in C^{2}[0,1]$. For $g_{0}(x)=x$ we get $C_{2}=\sup _{x \in[0,1]}\left(k^{-1}(x)\right)^{\prime \prime}<1$.

Note that the arguments leading to the estimates (21) are very simple and quite elementary.

The application of Corollary 10 is possible but supplies a larger value for $q$. Moreover, the reasoning being the basis of Corollary 10 is more complicated.

The estimate (21)/a) is an improved version of Kuzmin's celeḅrated theorem, cf., e.g., Khintchine [10: p. 76]. Kuzmin has given the remainder $O\left(q^{\sqrt{n}}\right)$. The improved remainder in (21)/a) is due to Lévy who found it independently of Kuzmin and only a bit later. Many years later the constant $q$ was improved by Szüsz [15] to $q<0.4$, and again later Wirsing [16] has found that $q<0.3037$ and that this estimate is not improvable. For a survey on the history of the problem cf. also Iosifescu [4].

\section{Piecewise linear transformations}

In the following we consider transformations $h$ for which

$$
h_{i}(x)=x_{i}+\left(y_{i}-x_{i}\right) x .
$$

We assume $x_{i+1}=y_{i}$ for all $i$, i.e., the $x_{i}$ can accumulate at most in the right end of the unit interval. All transformations $h$ defined by (22) preserve the uniform distribution. If $h$ is given by (22), then we can relax the suppositions on $g_{0}$.

Theorem 13: Let $g_{0}$ be concave on $[0,1)$ and assume that $g_{0}^{\prime}(0)$ and $g_{0}^{\prime}(1)$ exist. Then we have the non-uniform estimate

$$
\left|g_{1}(x)-x\right| \leq q x(1-x)\left(g_{0}^{\prime}(0)-g_{0}^{\prime}(1)\right), \text { where } q=\max _{i}\left(\left|x_{i}-x_{i+1}\right|\right)
$$

Proof: We estimate

$$
S=g_{1}(x)-x=\sum_{i=1}^{\infty}\left(g_{0}\left(h_{i}(x)\right)-g_{0}\left(x_{i}\right)-x\left(g_{0}\left(x_{i+1}\right)-g_{0}\left(x_{i}\right)\right)\right) .
$$


For $i>N$ we replace $x_{i}$ by 1 . Moreover we choose an integer $m_{1}$ and replace $x$ by $r / m_{1}$ and $x_{i}$ by $r_{i} / m_{1}$ for $i \leq N$, where $r$ and $r_{i}$ are integers and where the $r_{i}$ are increasing. Then $S$ changes over to $S_{m_{1}}$ where $\left|S-S_{m_{1}}\right| \leq \epsilon$. This is possible if we choose first $N$ and then $m_{1}$ large enough. We set $m=m_{1}^{2}$ and write $S_{m_{1}}=\sum_{n=1}^{m} a_{n} b_{n}$, where $b_{n}=g_{0}(n / m)-g_{0}((n-1) / m)$ and where

$$
a_{n}=\left\{\begin{array}{lll}
1-r / m_{1} & \text { for } & r_{i} m_{1}<n \leq r_{i} m_{1}+\left(r_{i+1}-r_{i}\right) r \\
-r / m_{1} & \text { for } & r_{i} m_{1}+\left(r_{i+1}-r_{i}\right) r<n \leq r_{i+1} m_{1}
\end{array}\right.
$$

We put $A_{n}=\sum_{k=1}^{n} a_{k}$ and get

$$
A_{r_{i} m_{1}}=0, A_{m}=0 \quad \text { as well. } \quad \text { as } 0 \leq A_{n} \leq R=r\left(1-r / m_{1}\right) \max _{i}\left(r_{i+1}-r_{i}\right) .
$$

In $S_{m_{1}}=\sum a_{n} b_{n}$ we apply summation by parts and obtain

$$
\begin{aligned}
S_{m_{1}} & =\sum_{n=1}^{m-1} A_{n}\left(b_{n}-b_{n+1}\right) ; \\
\left|S_{m_{1}}\right| & \leq R \sum_{n=1}^{m-1}\left(b_{n}-b_{n+1}\right) \leq R\left(b_{1}-b_{m}\right) \\
& =R\left(g_{0}(1 / m)-g_{0}(0)-g_{0}(1)+g_{0}((m-1) / m)\right) \rightarrow q x(1-x)\left(g_{0}^{\prime}(0)-g_{0}^{\prime}(1)\right)
\end{aligned}
$$

as $m_{1} \rightarrow \infty$. This proves our assertion as $\epsilon \rightarrow 0$ I

Corollary 14: Under the suppositions of Theorem 13 we have

$$
\left|g_{n}(x)-x\right| \leq q^{n} x(1-x)\left(g_{0}^{\prime}(0)-g_{0}^{\prime}(1)\right)
$$

Proof: If $q$ is the maximal length of the intervals for $h$, then $q^{n}$ is the maximal length of the intervals for $h^{\text {on }}$

We remark that (24) can be generalized if $g_{0}$ is concave for $b_{2 i} \leq x \leq b_{2 i+1}$ and convex for $b_{2 i+1} \leq x \leq b_{2 i+2}, i=1,2, \ldots, N$. Then

$$
\left|g_{n}(x)-x\right| \leq q^{n} x(1-x) \sum_{i=1}^{N} \gamma_{i}
$$

where

$$
\gamma_{i}=g_{0}^{\prime}\left(b_{2 i}+0\right)-g_{0}^{\prime}\left(b_{2 i+1}-0\right)-g_{0}^{\prime}\left(b_{2 i+1}+0\right)+g_{0}^{\prime}\left(b_{2 i+2}-0\right) .
$$

In this connection $b_{i}=b_{i+1}$ is allowed. Corollary 14 generalizes Theorem 3 in Goh and Schmutz [2]. We remark further that the assertions of Theorem 13 and Corollary 14 are valid if the $x_{i}$ have a finite number of limit points in the unit interval or if the number of limit points is arbitrary and $g_{0}$ is absolutely continuous.

Again we consider $h=k^{-1} \circ h^{*} \circ k$, where $h^{*}$ is defined by (22), i.e.,

$$
h_{i}(x)=k^{-1}\left(x_{i}+\left(y_{i}-x_{i}\right) k(x)\right) \text {. }
$$

Corollary 15 : Let the transformation $h$ be given by (25), let $g_{0}$ fulfil the suppositions of Theorem 13. Further assume that $k$ is convex and that $k^{\prime}(0), k^{\prime}(1)>0$. Then

$$
\left|g_{n}(x)-k(x)\right| \leq q^{n} k(x)(1-k(x))\left(g_{0}^{\prime}(0) / k^{\prime}(0)-g_{0}^{\prime}(1) / k^{\prime}(1)\right)
$$


Proof: By supposition $k^{-1}$ is concave and consequently $g_{0}^{*}=g_{0} \circ k^{-1}$ is concave, too. Furthermore, $g_{0}^{* \prime}(0)=g_{0}^{\prime}(0) / k^{\prime}(0)$ and $g_{0}^{* \prime}(1)=g_{0}^{\prime}(1) / k^{\prime}(1)$. It follows that $\left|g_{n}^{*}(x)-x\right| \leq$ $q^{n} x(1-x)\left(g_{0}^{\prime}(0) / k^{\prime}(0)-g_{0}^{\prime}(1) / k^{\prime}(1)\right)$ which implies (26)

Corollary 15 generalizes and improves Theorem 1 in Goh and Schmutz [2].

Example 16: We consider the functions $h$ and $k$ of Example 12. But we replace $\left(h^{*}\right)^{\text {on }}$ by a piecewise linear function $H_{n}^{*}$ with the same partition of $[0,1)$ as $\left(h^{*}\right)^{o n}$, i.e.,

$$
H_{n}^{*}(x)= \begin{cases}\left(x-h^{*}\left(j_{n} ; 0\right)\right) / \Delta_{j_{n}} & \text { for } h^{*}\left(j_{n} ; 0\right) \leq x<h^{*}\left(j_{n} ; 1\right), \\ \left(x-h^{*}\left(j_{n} ; 1\right)\right) / \Delta_{j_{n}} & \text { for } h^{*}\left(j_{n} ; 1\right) \leq x<h^{*}\left(j_{n} ; 0\right),\end{cases}
$$

where $\Delta_{j_{n}}=\left|h^{*}\left(j_{n} ; 1\right)-h^{*}\left(j_{n} ; 0\right)\right|$. Note that $H_{n}^{*}$ is different from $\left(H_{1}^{*}\right)^{n}$. We have

$$
h^{*}\left(j_{n} ; x\right)=\frac{1}{\log 2} \log \left(1+\frac{p_{n}+p_{n-1}(t-1)}{q_{n}+q_{n-1}(t-1)}\right) \text {, }
$$

cf. Khintchine [10: p.79]. Here $p_{n}$ and $q_{n}$ are the numerator and the denominator, respectively, of the $n$-th convergent $p_{n} / q_{n}=\left[i_{1}, i_{2}, \ldots, i_{n}\right]$ formed with the partial quotients $i_{1}, i_{2}, \ldots, i_{n}$. It follows that

$$
\log 2 \cdot \Delta_{j_{n}}=\left|\log \left(1+\frac{p_{n}+p_{n-1}}{q_{n}+q_{n-1}}\right)-\log \left(1+\frac{p_{n}}{q_{n}}\right)\right|=\left|\log \left(1+\frac{(-1)^{n}}{\left(q_{n}+p_{n}\right)\left(q_{n}+q_{n-1}\right)}\right)\right| .
$$

The maximum of $\Delta_{j_{n}}$ is achieved for $j_{n}=(1, \ldots, 1)$. In this case we have $p_{n}=f_{n}, q_{n}=f_{n+1}$, where $f_{n+2}=f_{n+1}+f_{n}, f_{1}=f_{2}=1$ is the sequence of Fibonacci numbers. It is well-known that $f_{n} \sim((1+\sqrt{5}) / 2)^{n} / \sqrt{5}$ as $n \rightarrow \infty$. We put $H_{n}=k^{-1} \circ H_{n}^{*} \circ k$ and obtain $\left|H_{n}(x)-k(x)\right|<C q^{n}$, for $q=2 /(3+\sqrt{5}) \leq 0.3820$.

- In the following last two theorems let $h$ be given by (22), but $x_{i+1}=y_{i}$ for all $i$ is not needed, i.e., the $x_{i}$ can have an arbitrary number of limit points in the unit interval.

Theorem 17: If $g_{0} \in C^{2}[0,1)$, then $\left|g_{n}^{\prime}(x)-1\right| \leq q^{n} \sup _{x \in[0,1]}\left|g_{0}^{\prime}(x)\right|$ :

Proof: We have

$$
g_{1}^{\prime}(x)-1=\sum_{i=1}^{\infty}\left(h_{i}^{\prime}(x) g_{0}^{\prime}\left(h_{i}(x)\right)-\int_{x_{i}}^{y_{i}} g_{0}^{\prime}(x) d x\right)=\sum_{i=1}^{\infty}\left(y_{i}-x_{i}\right)\left(g_{0}^{\prime}\left(h_{i}(x)\right)-g_{0}^{\prime}\left(\xi_{i}\right)\right)
$$

a.e. and consequently $\left|g_{1}^{\prime}(x)-1\right| \leq q \sup _{x \in[0,1)}\left|g_{0}^{\prime \prime}(x)\right|$. Now the assertion follows by considering, $h^{\text {on }}$ instead of $h$ 】

Theorem 18: If $g_{0} \in C^{k}[0,1], k \geq 2$, then $\left|g_{n}^{(k)}(x)\right|<q^{(k-1) n} \sup _{x \in[0,1)}\left|g_{0}^{(k)}(x)\right|$.

Proof: We differentiate (2) and obtain

$$
g_{1}^{(k)}(x)=\sum_{i=1}^{\infty}\left(h_{i}^{\prime}(x)\right)^{k} g_{0}^{(k)}\left(h_{i}(x)\right) \text { and }\left|g_{1}^{(k)}(x)\right| \leq q^{k-1} \sup _{x \in[0,1)}\left|g_{0}^{(k)}(x)\right| \sum_{i=1}^{\infty} h_{i}^{\prime}(x)
$$

which implies the assertion

Example 19: Let $g_{0}(x)=x+\beta x(1-x)$, where $-1 \leq \beta \leq 1$. Then $g_{0}^{\prime \prime}(x)=-2 \beta$ and therefore $g_{1}^{\prime \prime}(x)=-2 \beta q, q=\sum_{i=1}^{\infty}\left(h_{i}^{\prime}(x)\right)^{2}=\sum_{i=1}^{\infty}\left(y_{i}-x_{i}\right)^{2}<1$ in view of (27). We obtain $g_{n}^{\prime \prime}(x)=-2 \beta q^{n}$ which implies $g_{n}(x)=x+\beta q^{n} x(1-x)$.

Acknowledgement: The author is very indebted to $\mathrm{L}$. Heinrich for the references to $[3-5,12]$ and for the proof of (16) which was formerly an additional supposition in Theorem 9. Furthermore, the author thanks one referee for many useful hints. 


\section{REFERENCES}

[1] Bosch, W.: Functions that preserve the uniform distribution of sequences. Trans. Amer. Math. Soc. 307 (1988), 143 - 152.

[2] Goh, W. M. Y. and E. Schmutz: Universal asymptotic distribution functions mod 1. Lect. Notes Pure Appl. Math. 124(1990),,619 - 626.

[3] Hofbauer, F. and G. Keller: Ergodic properties of invariant measures for piecewise monotonic transformations. Math. Z. 180(1982), $119-140$.

[4] Iosifescu, M.: A survey of the metric theory of continued fractions, fifty years after Doeblin's 1940 paper. In: Probability theory and mathematical statistics (Proc. V. Vilnius Conf. Vilnius/Lith. 1989, eds.: B. Grigelonis, Yu. V. Prokhorov, V.V. Sazonov, V. Statulevičius). Vol. 1 (1990), $550-572$.

[5] Ishitani, H.: A central limit theorem of mixed type for a class of 1-dimensional transformations. Hiroshima Math. J. 16 (1986), 161 - 188.

[6] Jabloniski, M.: On the convergence of iterates of the Frobenius-Perron operator for expanding mappings. Ann. Pol. Math. 48 (1988), 127 - 130.

[7] Jabloński, M., Kowalski, Z. S., and J. Malczak: The rate of convergence of iterates of the FrobeniusPerron operator for Lasota-Yorke transformations. Univ. Iagell. Acta Math. 25 (1985), 189 - 193.

[8] Jabloniski, M. and J. Malczak: The rate of convergence of iterates of the Frobenius-Perron operator for piecewise convex transformations. Bull. Polish Acad. Sci. Math. 31(1983), $249-254$.

[9] Jabloński, M. and J. Malczak: The rate of convergence of the Frobenius-Perron operator. Colloq Math. 49 (1984), $67-72$.

[10] Khintchine, A.: Kettenbrüche. Leipzig: B.G. Teubner Vertragsgesellschaft 1956.

[11] Kuipers, L. and H. Niederreiter: Uniform distribution of sequences. New York: Wiley 1974.

[12] Lasota, A. and J. A. Yorke: On the existence of invariant measures for piecewise monotonic transformations. Trans. Amer. Math. Soc. 186 (1973), $481-488$.

[13] Lasota, A. and J. A. Yorke: Exact dynamical systems and the Frobenius-Perron operator. Trans. Amer. Math. Soc. 273 (1982), 375 - 384.

[14] Porubsky, S., Šalát, T., and O. Strauch: Transformations that preserve uniform distribution. Acta Arith. 49 (1988), $459-479$.

[15] Szüsz, P.: Über einen Kusminschen Satz. Acta Math. Acad. Sci. Hungary 12 (1961), 447 - 453.

[16] Wirsing, E.: On the theorem of Gauss-Kusmin-Lévy and a Frobenius-type theorem for function spaces. Acta Arith. 24 (1974), 507 - 528. 